\title{
DIAMONDS FROM THE EKATI CORE AND BUFFER ZONE PROPERTIES
}

\author{
John Gurney ${ }^{1}$, Peter Hildebrand ${ }^{1}$, Jon Carlson ${ }^{2}$, Darren Dyck ${ }^{2}$ and Yana Fedortchouk ${ }^{2}$ \\ ${ }^{1}$ Mineral Services, South Africa; ${ }^{2}$ BHP Billiton Diamonds Inc. Canada
}

\section{INTRODUCTION}

Approximately 150 kimberlite occurrences have been confirmed in the Ekati Mine core and buffer zone properties. Nearly one quarter of these known pipes have been tested for macrodiamonds and large variations in the appearance of parcels of diamonds from individual localities are common. In detail the differences can be almost entirely accounted for by variations in the proportions of sub-populations of diamonds, termed components. These components characterized on the basis of visual similarity and influenced by appropriate recognition of well established processes relevant to diamond formation and preservation. At present, seven such major and five minor components have been observed. The major groups incorporate white, brown and fibrous diamonds with other distinguishing features. Published analyses of included minerals in diamonds, together with FTIR analysis of diamonds have established at least five diamond forming and modifying processes within the Slave craton. Observational evidence suggests that there are even more.

The overall characteristics for run of mine diamonds at a particular locality at Ekati reflect the efficacy with which the kimberlite has sampled separate zones of preexisting diamond bearing rocks within the craton root, sometimes with modification during transport to the surface by resorption. For the major groupings where enough diamonds have been recovered to establish a size distribution pattern with some certainty, these clearly vary from component to component. A similar situation can be expected to apply to the minor subpopulations of diamonds as well as the larger ones.

\section{OBSERVATIONS}

Comparison of diamond parcels from localities within the Ekati mine demonstrate that the prevailing characteristics of each locality can be largely defined in the context of the proposed model, even where there are major apparent differences. At Ekati such situations sometimes arise between kimberlites of similar age and close geographic association, for example Beartooth and Grizzly. (Locations of Ekati kimberlites shown in Figure 1).

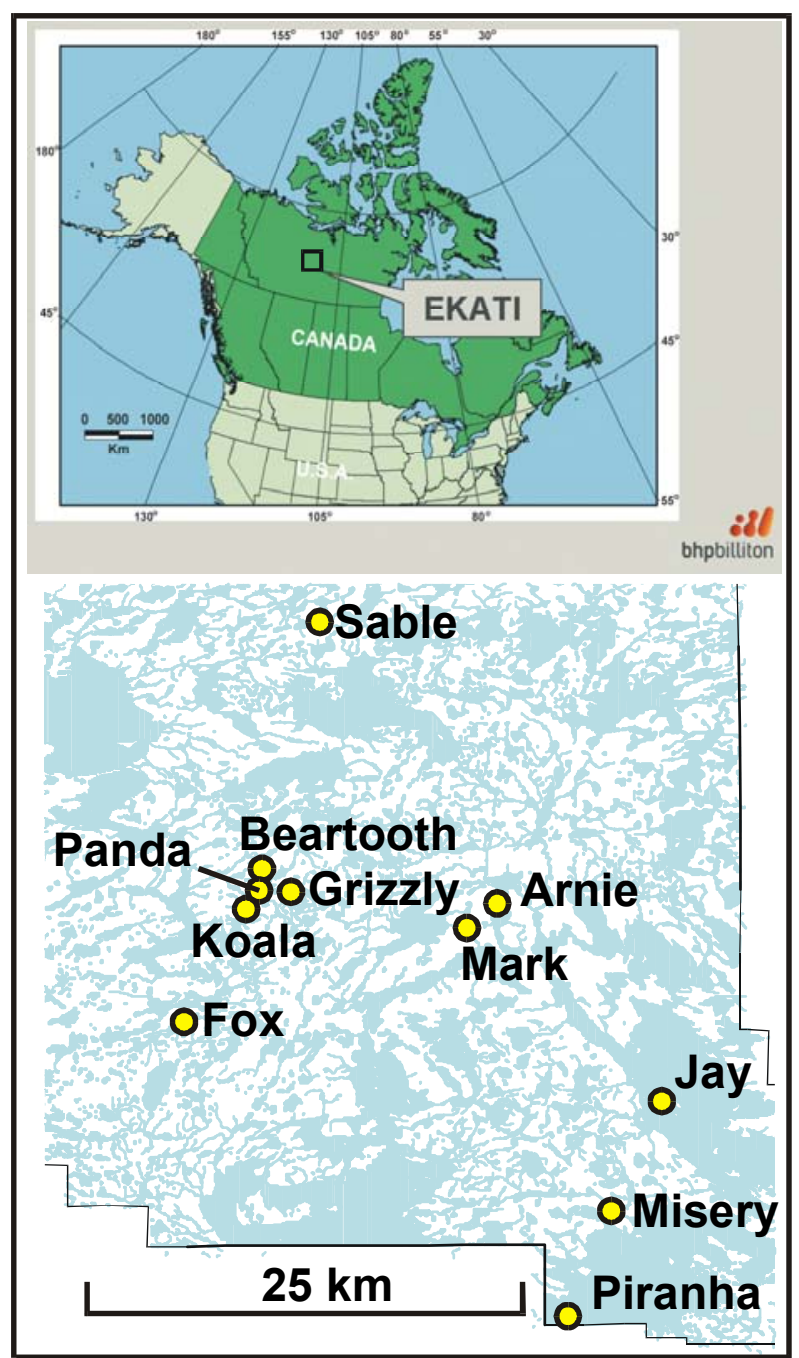

Figure 1: Locations of selected Ekati kimberlites.

At Beartooth (Figure 2) colourless octahedra predominate and coated octahedra are commonly accompanied by colourless macles, brown octahedra and fibrous cubic diamonds. Dodecahedra of any colour are very rare. Less than $1 \%$ of the diamonds are lemon yellow cubes and dodecahedra. Similar characteristics are seen at the adjacent kimberlites Panda and Koala. 

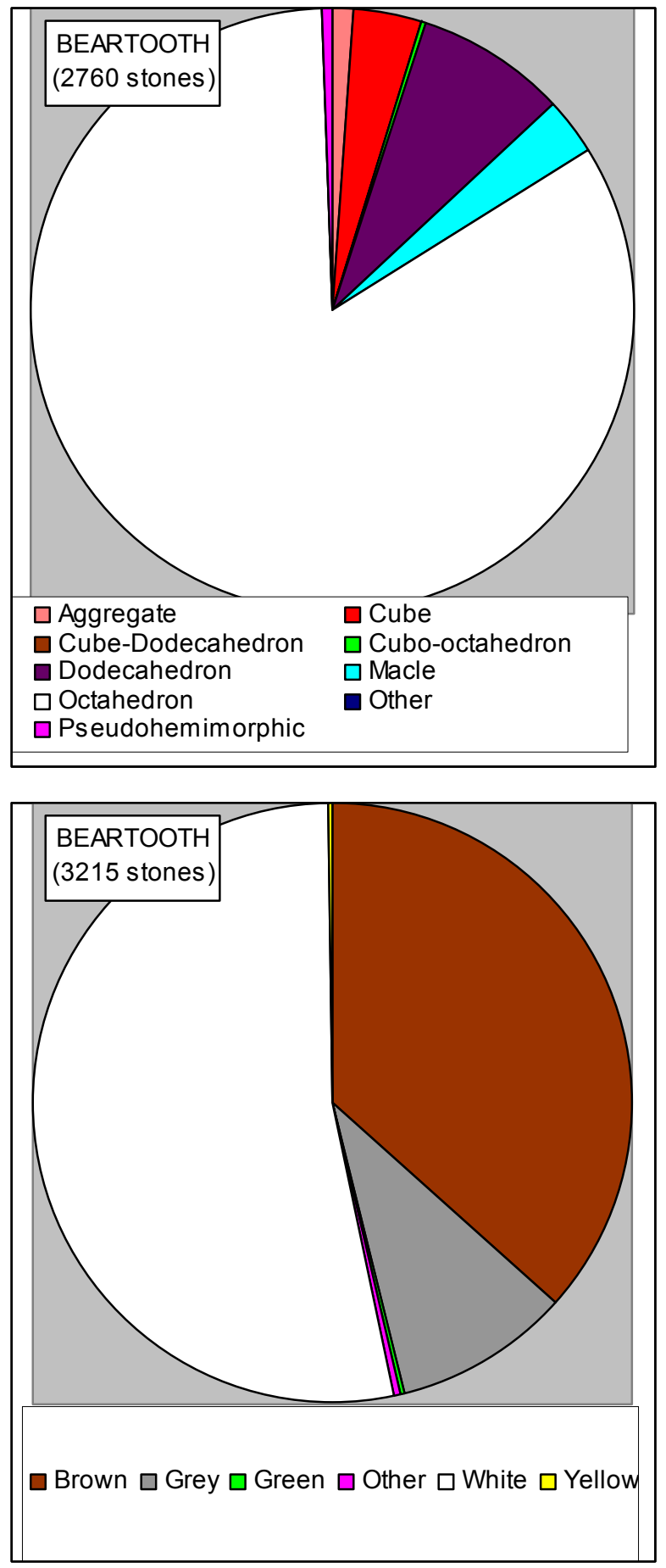

Figure 2: Beartooth kimberlite proportions of stones by morphology and colour. The predominantly octahedral and white followed by brown stones is also a feature of nearby Panda and Koala, but not Grizzly.

At Grizzly (Figure 3) similar components are seen in the run of mine production. However brown is the predominant diamond colour in contrast to the high
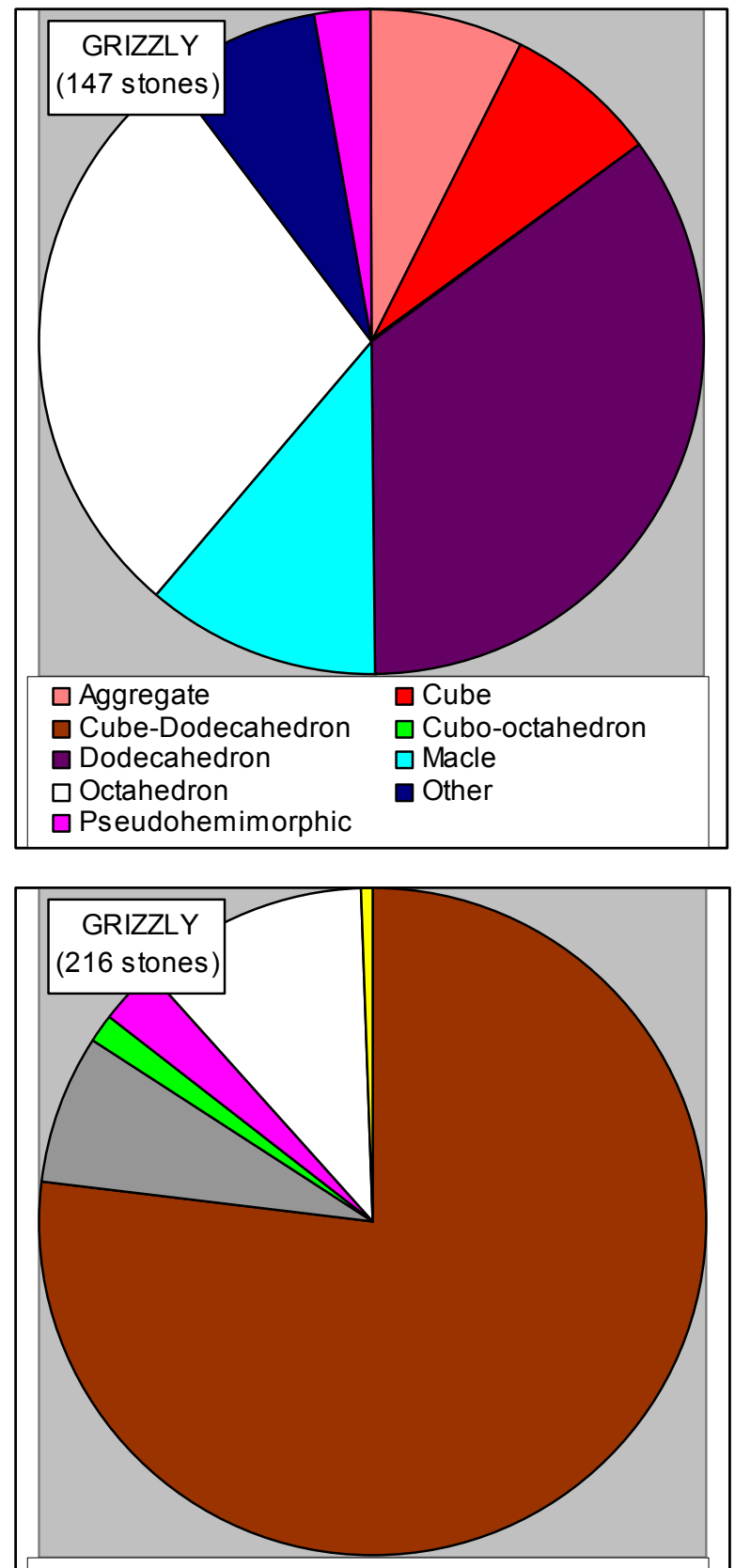

Brown $\square$ Grey $\square$ Green $\square$ Other $\square$ White $\square$ Yellow

Figure 3: Grizzly proportions of stones by morphology and colour. The strong brown dominance replaces the colourless stones from localities like Beartooth. The brown diamonds from Grizzly fall into a number of significant morphologies, notably octahedral, macles and dodecahedra. This is a marked contrast to Beartooth (shown in Figure 1).

abundance of colourless octahedral diamonds at Beartooth and Panda. More abundant dodecahedra, 
macles, aggregates and cubes compensate for the lower abundance of the octahedra.

Again comparing the Misery Kimberlite to Beartooth and Panda, the diamond population contains a similar range of diamond shapes, colours and surface features, but in differing proportions. Dodecahedra predominate over octahedra in both colourless and brown diamonds. Brown diamonds are more common than colourless, the lemon yellow diamonds rarely seen at Beartooth are more abundant though still a minor feature in the Misery production. Cubes and fibrous coats are very rare.

The predominance of brown diamonds is even more pronounced at Jay where the majority of browns are dodecahedra. Colourless diamonds make up $\sim 20 \%$ of the overall population with a heavy predominance of dodecahedra. Yellow is an extremely rare colour, whilst fibrous cubes form less than $0.1 \%$ of the overall population, and coated diamonds are also rare.

Both the Arnie and Mark Kimberlites provide a further variation in observed abundances of different stone categories. Colourless octahedra are rare and small. Colourless dodecahedra more abundant but also small. Brown diamonds, predominantly dodecahedra, account for $\sim 10 \%$ by weight of the diamond population which is completely dominated by opaque fibrous cubes and fragments.

\section{FINAL REMARKS}

Considering all diamonds recovered from these localities, the major observed differences are the result of variations in the proportions of eleven components: colourless flat faced sharp edged octahedral (Figure 4), colourless step faced octahedral (Figure 5), coated white octahedral (Figure 6), colourless macles, colourless dodecahedra (Figure 7), light brown rounded resorbed octahedra (Figure 8), pale brown dodecahedra (Figure 9), dark brown octahedra (Figure 10), dark brown dodecahedra (Figure 11), minor lemon yellow cubes and dodecahedra (Figure 12) and fibrous cubes (Figure 13). These observations can be extended to throughout the Ekati property with the introduction of an additional cubo-octahedral category, particularly at Piranha.

Some of these components may have formed in the same process but this is highly unlikely for all. The reality may be similar to Letseng la Terai where McDade and Harris (1999) recognised 10 distinct growth events from 4 distinct lithologies in a low-grade ore body.

\section{REFERENCE}

McDade, P., Harris, J.W., 1999. Syngenetic Inclusion bearing diamonds Letseng-la-Terai, Lesotho. In: Gurney, J.J., Gurney, J.L., Pascoe, M.D., Richardson, S.H., (Eds.), P. H. Nixon volume, Proceedings of the 7th International Kimberlite Conference, Red Roof Design, Cape Town, 557-565.

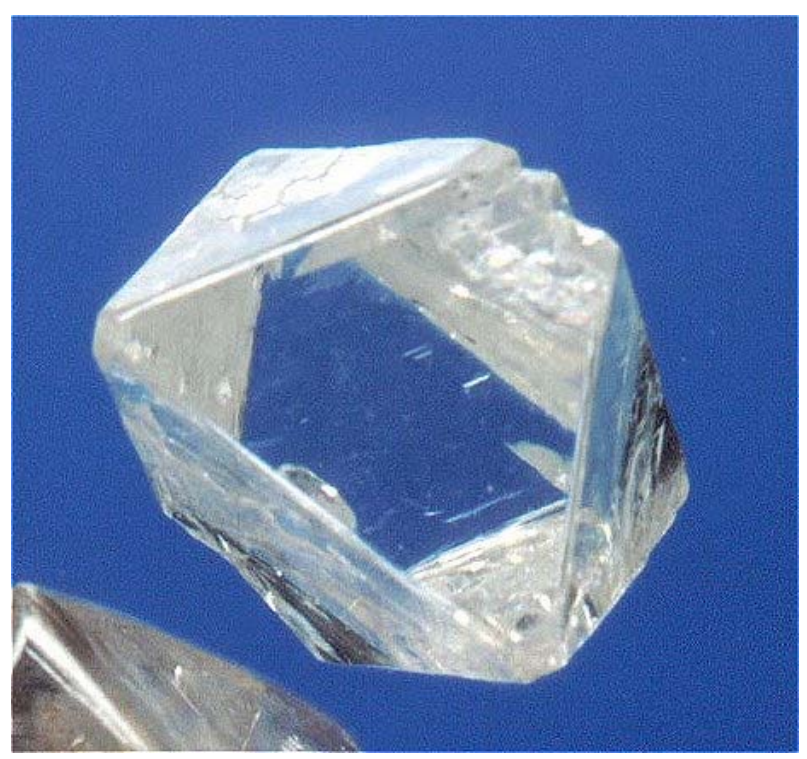

Figure 4: Colourless flat faced sharp edged octahedron with an imperfect termination typifying this component on the Ekati property. Observations of rare inclusions in these diamonds suggest a peridotitic origin. This diamond is from the Fox kimberlite. Similar diamonds are an important component in the better quality diamond populations from such pipes as Panda and Koala. 


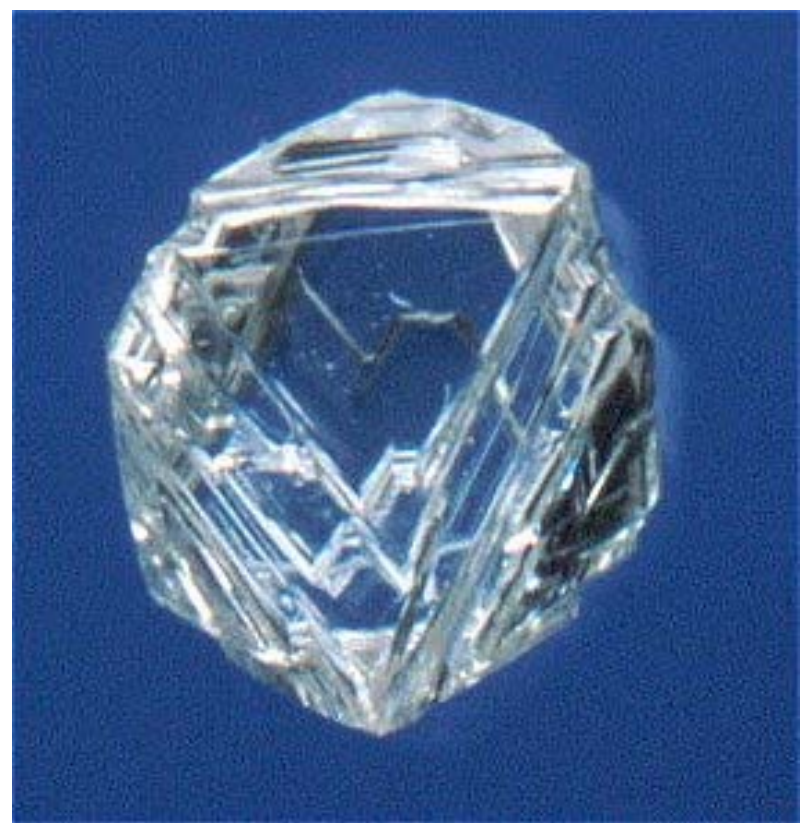

Figure 5: Colourless step faced octahedron from the Koala kimberlite. Generally subordinate in abundance to the flat-faced octahedra shown in Figure 3.

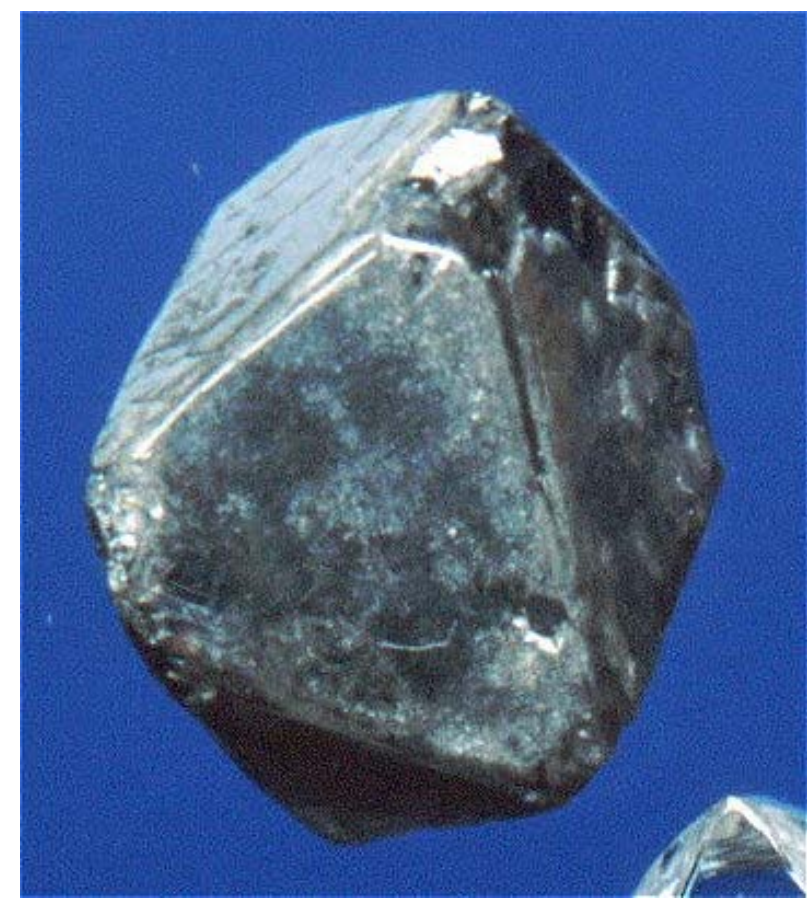

Figure 6: Octahedron with a younger fibrous diamond coat. The octahedral nucleus, where observable, is generally white and well preserved from resorbtion. The coat varies in thickness from up to $2 \mathrm{~mm}$ to barely visible. Diamond from the Panda mine.

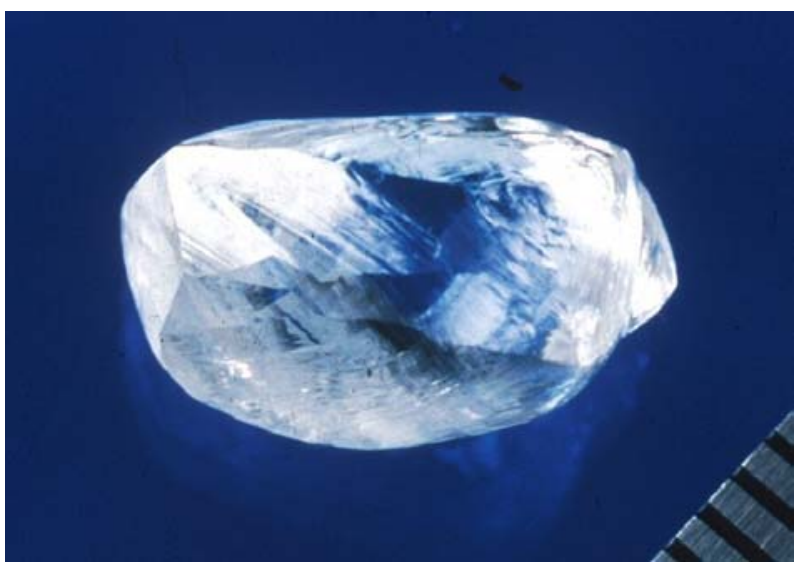

Figure 7: Heavily resorbed rounded colourless dodecahedron of excellent quality from Misery mine.

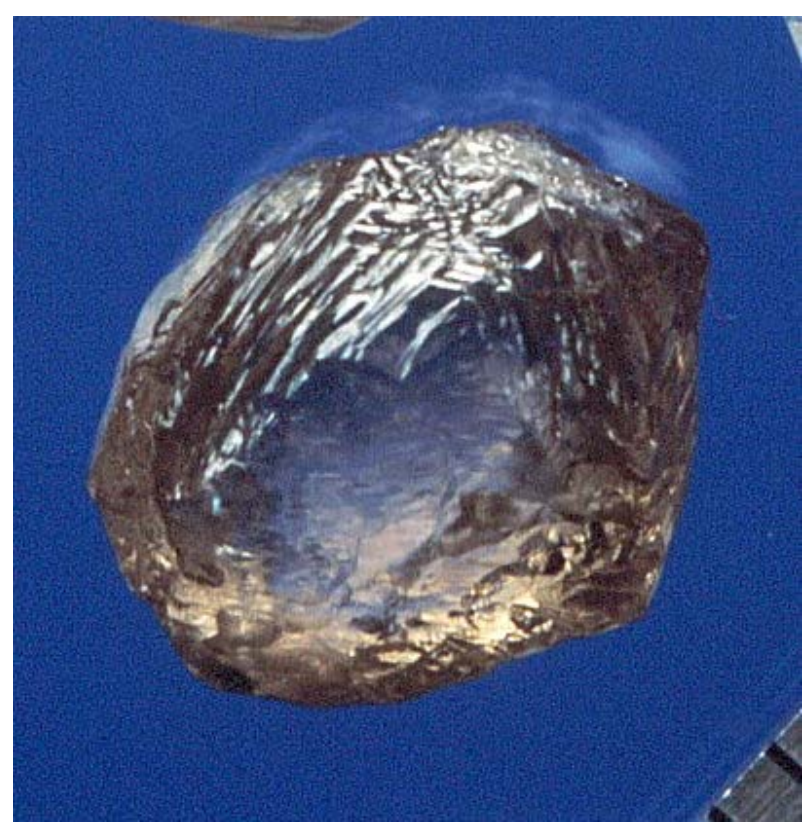

Figure 8: Light brown rounded resorbed octahedron from the Fox kimberlite.

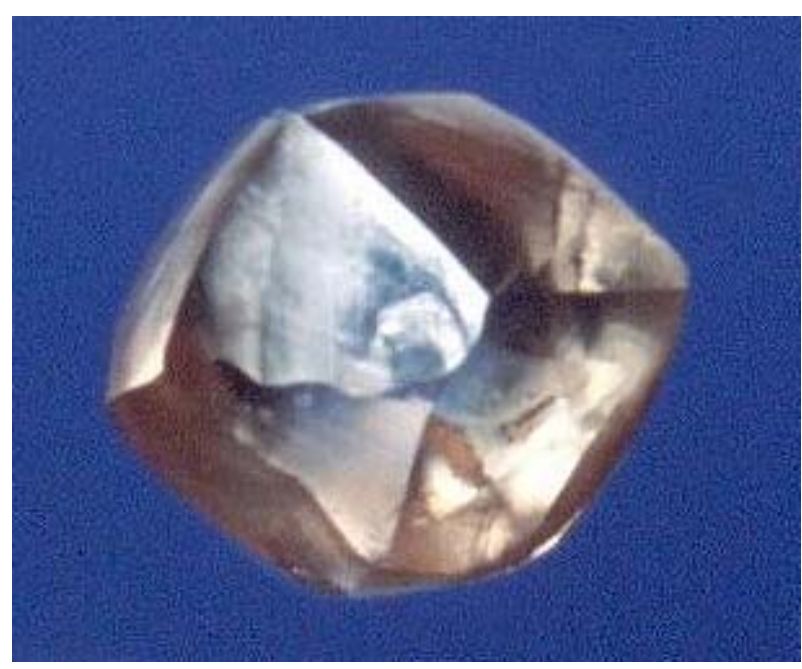

Figure 9: Light brown dodecahedron from the Misery mine, where brown resorbed diamonds predominate. 


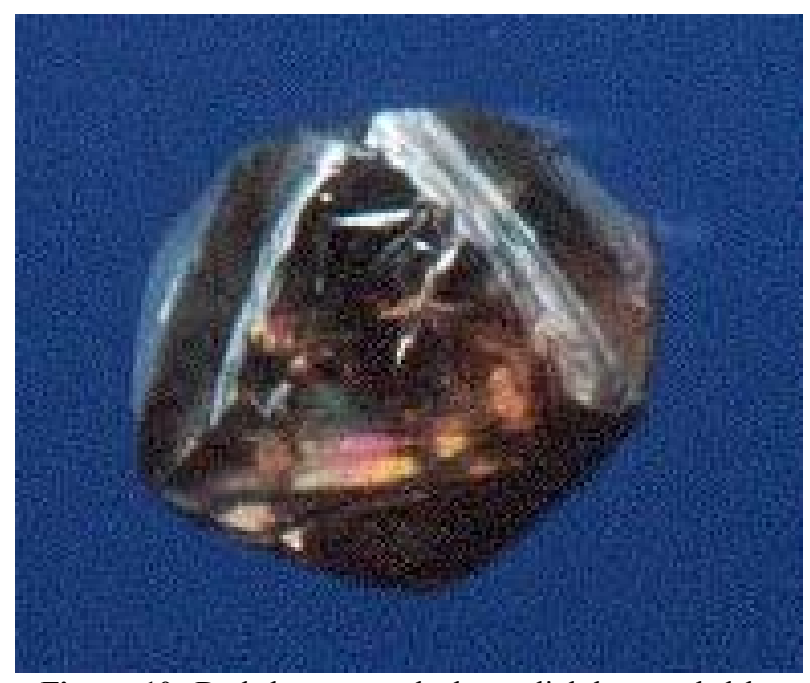

Figure 10: Dark brown octahedron, slightly rounded by resorption from the Koala kimberlite. Common at Misery and Jay.

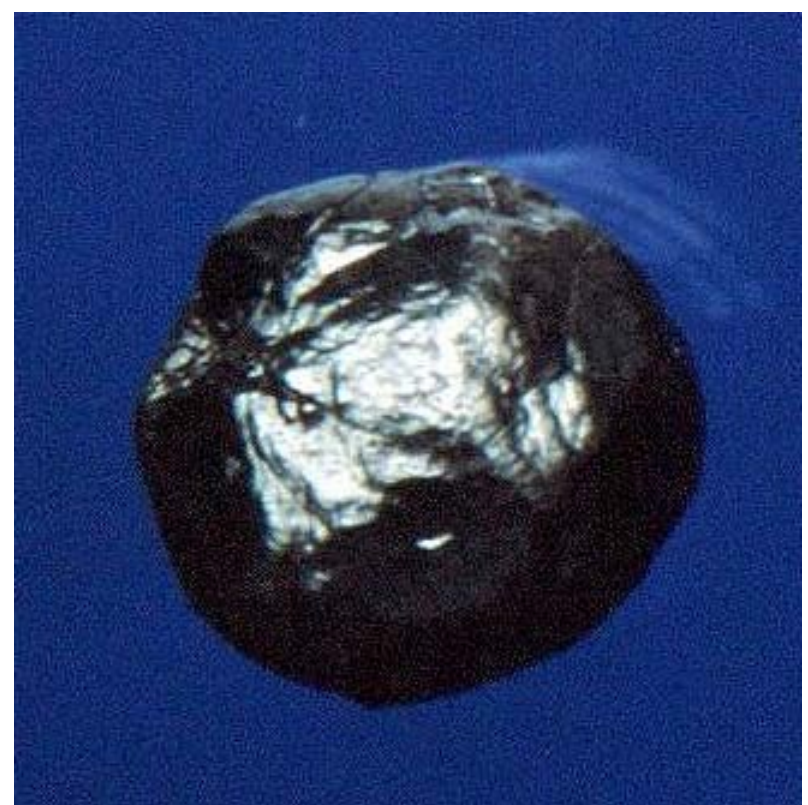

Figure 11: Dark brown, very rounded, highly resorbed and rutted diamond from the Sable kimberlite. Very rare at Panda, Beartooth and Koala. More abundant at Misery and Jay.

Contact: JJ Gurney, PO Box 38668, Pinelands, Cape Town, South Africa 7430, E-mail: John.Gurney@minserv.co.za

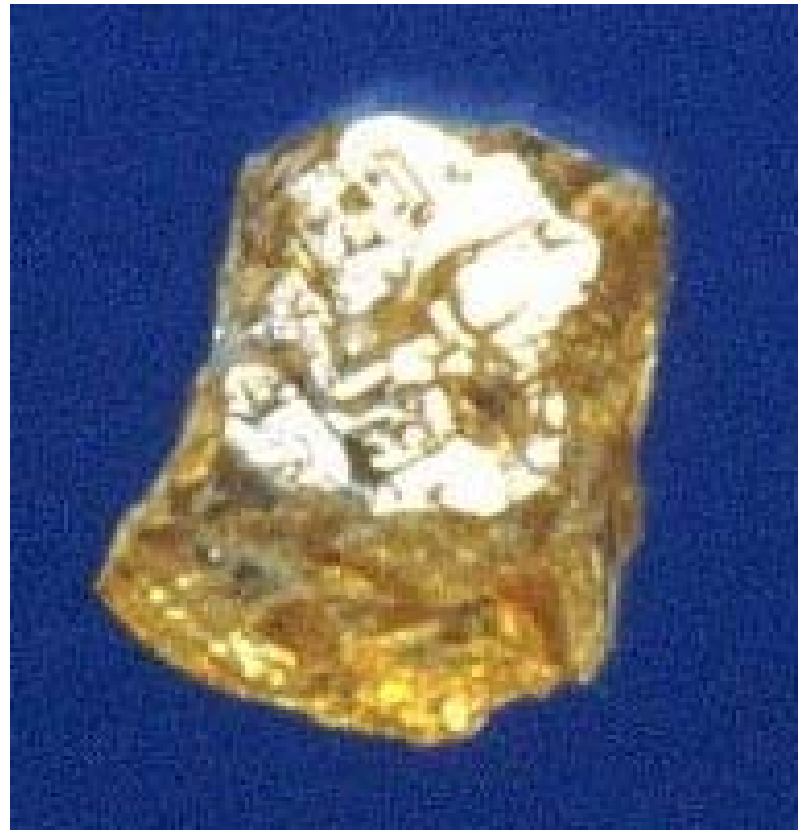

Figure 12: Yellow cube from the Sable kimberlite. A minor but persistent component of Ekati diamond populations.

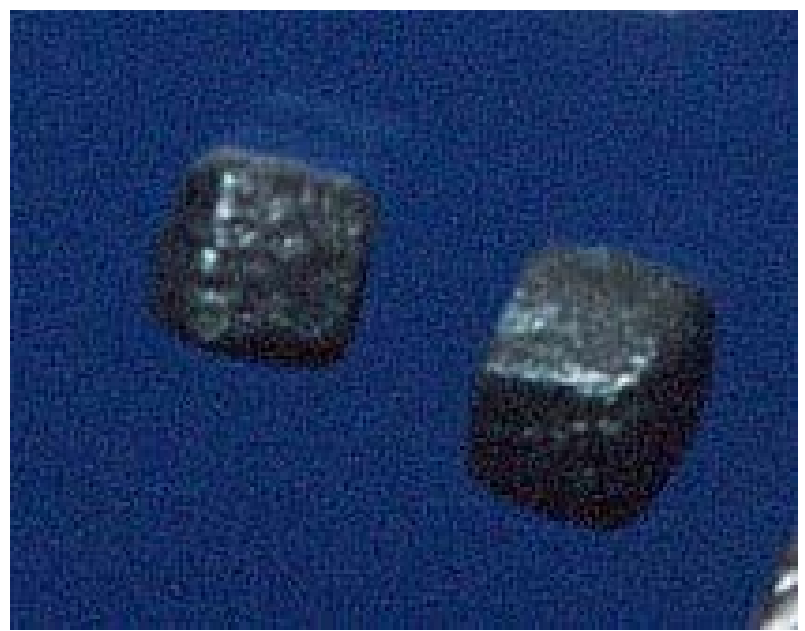

Figure 13: Two fibrous cubes from the Koala kimberlite. Possibly linked to the fibrous diamond coats seen on some Ekati octahedra, these fibrous diamonds are very rare at some localities (e.g. Jay, Misery), a significant component at others (e.g. Koala) and very occasionally dominant (Mark, Arnie). They are predominantly small $(<0.1 \mathrm{ct} /$ stone, very rarely $>0.2 \mathrm{ct} /$ stone). 